\title{
Resonant Raman-active acoustic phonons in the mixed amorphous-microcrystalline phase of ion-implanted GaAs
}

\author{
M. Holtz ${ }^{*}$ and R. Zallen \\ Department of Physics, Virginia Tech, Blacksburg, Virginia 24061 \\ O. Brafman \\ Department of Physics and Solid State Institute, Technion-Israel Institute of Technology, Haifa, Israel
}

(Received 21 September 1987)

\begin{abstract}
We have observed a new, strong, low-frequency peak (at $47 \mathrm{~cm}^{-1}$ ) in the Raman spectrum of ion-implanted GaAs having a mixed amorphous-microcrystalline microstructure. It is strongly resonant near $1.7 \mathrm{eV}$, just above the band gap, in contrast to the longitudinal-optic phonon line of the microcrystals (which resonates differently) and the bands of the amorphous component (which do not resonate). We tentatively interpret this peak in terms of acoustic phonons made Raman active by the presence of microcrystal-amorphous interface regions, and discuss several models.
\end{abstract}

\section{INTRODUCTION}

Ion implantation provides a means by which crystalline GaAs, $c-G a A s$, can be converted to a material composed of microcrystals mixed with the amorphous form, $a$ GaAs. ${ }^{1-3}$ As crystallites become very small, long-range order is lost and finite-size effects become important. When examined using Raman scattering, the microcrystalline component of the ion-implanted "damage layer" manifests itself in the broadening and shifting of the $c$ GaAs spectral features. ${ }^{3,4}$ Simultaneously, the broad spectral signature of $a-\mathrm{GaAs}$ is observed.

In a recent article, ${ }^{3}$ we have reported Raman-scattering and chemical-etch experiments which reveal the implantation-induced formation of a near-surface region of uniform high damage (small crystallites, large $a$-GaAs volume fraction) in GaAs which has been bombarded with 45-keV Be ${ }^{+}$ions. This high-damage "plateau" extends to a depth of about $1500 \AA$. From these results we obtained the optical absorption coefficient $\alpha(\hbar \omega)$, within the plateau region of the implanted material, over a range of above-band-gap photon energies $(\hbar \omega)$ corresponding to various laser lines. Knowing $\alpha(\hbar \omega)$ enables us to separate the effect of photon-energy-dependent Ramanscattering efficiency from the effect of photon-energydependent scattering volume (optical penetration depth). Combining this with the fact that our experiments are probing a macroscopically uniform material (penetration less than the depth of the plateau), has enabled us to obtain the new resonance-Raman results which we report here.

In this Rapid Communication, we report a new, strong low-frequency peak in the Raman spectrum of implanted GaAs. This new peak, which resonates in the near infrared, is absent in the spectrum of $a-\mathrm{GaAs}$ and also has no counterpart in the vibrational density of states of $c$ GaAs. A tentative interpretation of this peak, which is evidently characteristic of the mixed amorphous-microcrystalline nature of ion-bombarded $\mathrm{GaAs}$, is given here. A more complete account of the experimental observations and theoretical models is in preparation. ${ }^{5}$

\section{EXPERIMENTAL RESULTS: RESONANT, IMPLANTATION-INDUCED, LOW- FREQUENCY RAMAN PEAK}

In Fig. 1 we show the Raman spectrum of (100)oriented $c-\mathrm{GaAs}$ and of implanted $\mathrm{GaAs}$ which has been bombarded by $45-\mathrm{keV} \mathrm{Be}^{+}$ions to various fluences (measured in units of ions $/ \mathrm{cm}^{2}$ ). No anneal was done on these

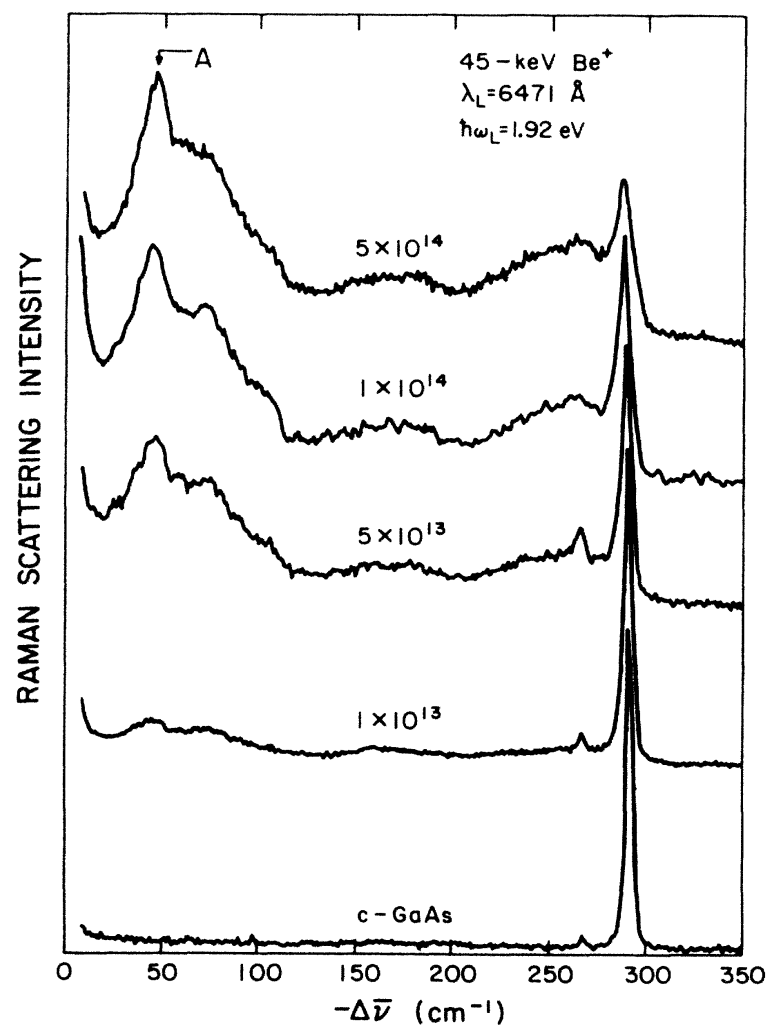

FIG. 1. Unpolarized, $300 \mathrm{~K}$, Raman spectra for Beimplanted GaAs samples, taken with $\hbar \omega_{L}=1.92 \mathrm{eV}$. Ion fluence is given in ions $/ \mathrm{cm}^{2}$. Intensities in Figs. 1-3 are normalized to a $\mathrm{CaF}_{2}$ standard. 
samples. Spectra were collected at $300 \mathrm{~K}$ in a nearbackscattering geometry under a flowing-argon atmosphere using 1.92-eV red light as excitation. Intensities are normalized to a $\mathrm{CaF}_{2}$ transparent standard. ${ }^{3}$ The $\mathrm{LO}$ line is seen to broaden and downshift in energy while decreasing in intensity with increasing ion fluence. Also, with increasing lattice damage, the broadband signature of $a$-GaAs increases in intensity. In addition, a new, strong feature $(A)$ is seen at $47 \mathrm{~cm}^{-1}$, which is not present in the crystal and which grows stronger with ion fluence.

Figure 2 shows the variation of the Raman spectrum, for the $5 \times 10^{14}-\mathrm{cm}^{-2}$ implant, as the photon energy of the exciting laser line is varied. For $\hbar \omega$ below $2 \mathrm{eV}$, feature $A$ is clearly observed; it is weak at higher photon energies, indicating strong resonance. The LO line shape does not vary, ${ }^{3,6}$ providing evidence that we are probing a region of uniform crystallite-size distribution. ${ }^{4}$ This has been confirmed by our chemical-etch depth-profile Raman studies, which show that the high-damage plateau in this material extends to about $1500 \AA$, and that the full damage layer extends to about $4000 \AA{ }^{3}$ For Raman spectra probing the structurally graded region beyond $1500 \AA$, the LO line becomes progressively sharper and more intense, while the $a-\mathrm{GaAs}$ bands weaken. But when probing depths less than $1500 \AA$, the spectral shape (for given $\hbar \omega)$ is depth independent. In the present paper, we are probing this region of fixed amorphous-microcrystalline

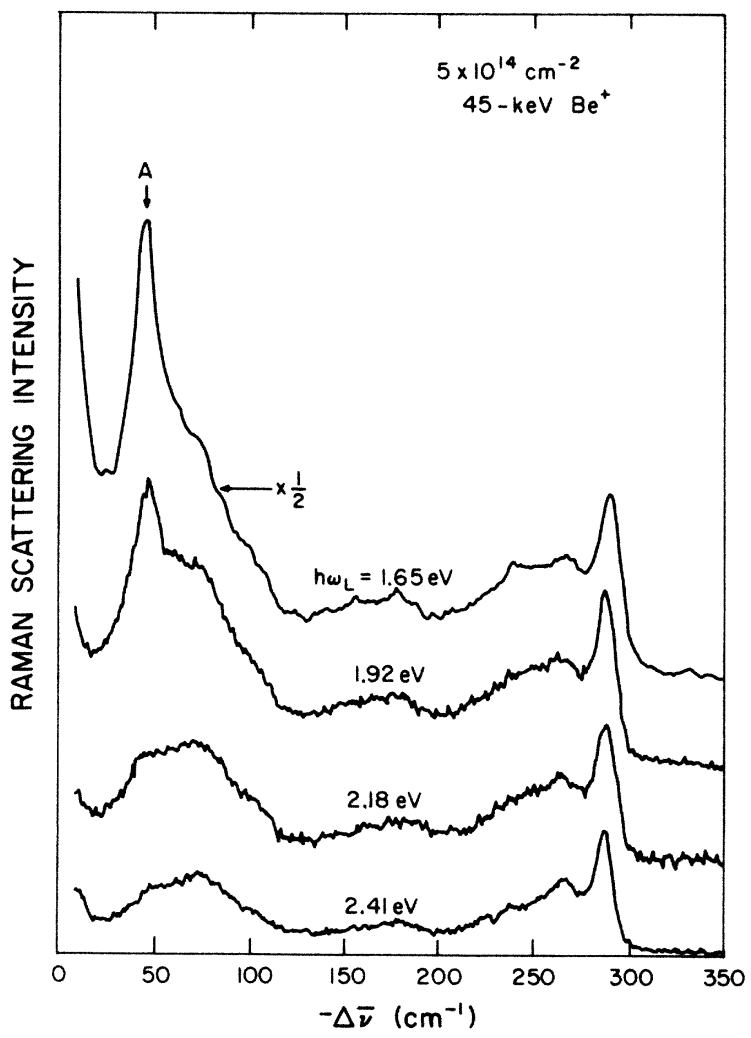

FIG. 2. Raman spectra for $5 \times 10^{14}-\mathrm{cm}^{-2}$ Be-implanted GaAs, for four values of $\hbar \omega_{L}$. The $1.65-\mathrm{eV}$ spectrum is scaled down by a factor of 2 . microstructure.

The points in Fig. 3 give the intensity (normalized to the $\mathrm{CaF}_{2}$ standard) of the $a-\mathrm{GaAs}$ component of the Raman spectrum of the $5 \times 10^{14}-\mathrm{cm}^{-2}$ implant. The $a-\mathrm{GaAs}$ intensity, $I_{a}$, is shown plotted against $\hbar \omega$ between 1.55 and $2.71 \mathrm{eV}$. Since amorphous GaAs has a featureless absorption spectrum in this region, ${ }^{7}$ any resonance effects should be quite weak and the $a-\mathrm{GaAs}$ Raman intensity system should be a good measure of scattering volume. In Ref. 3, we found that the experimental estimate obtained for the optical absorption spectrum, $\alpha(\hbar \omega)$, characteristic of the high-damage region of this implant, was well accounted for by the expression $\alpha(\hbar \omega)=f_{c} \alpha_{c}(\hbar \omega)$ $+f_{a} \alpha_{a}(\hbar \omega)$. Here, $\alpha_{c}$ and $\alpha_{a}$ are the respective absorption coefficients of $c$-GaAs (Ref. 8) and $a$-GaAs, ${ }^{1}$ while $f_{c}$ and $f_{a}$ are the volume fractions of the crystalline and amorphous components, obtained on the basis of the amorphous-microcrystalline model for the damage-layer microstructure. The curve plotted in Fig. 3 is the optical penetration depth, $d_{\mathrm{OPT}}=1 /(2 \alpha)$, corresponding to $f_{c} \alpha_{c}+f_{a} \alpha_{a}$ with $f_{c}=0.75$ and $f_{a}=0.25 .^{3}$ This curve (with vertical scale adjusted for overall fit) accounts very well for the photon-energy dependence of $I_{a}$. Thus $I_{a}$ serves as an internal spectral measure of $d_{\mathrm{OPT}}$ and the effective volume sampled with the use of a particular laser line. Normalizing another Raman band with respect to $I_{a}$ thus provides us with an estimate for the Ramanscattering efficiency of that band.

Figure 4 displays $I(\mathrm{LO})$ and $I(A)$ (both normalized to $I_{a}$ ) for the same sample, plotted versus laser photon energy. A strong $\hbar \omega$ dependence is seen for each. The LO line, which is Raman allowed for our geometry, has a constant scattering efficiency up to approximately $2.2 \mathrm{eV}$. Above $2.2 \mathrm{eV}$, the LO strength rapidly increases with increasing $\hbar \omega_{L}$. We interpret this as resonance with the $E_{1}$ interband electronic transition at $2.9 \mathrm{eV}$ in $c-G a A s .{ }^{9}$ The dashed curve in Fig. 4 is the calculated cross section for Raman-allowed scattering in resonance with the $E_{1}$ transition in $c$-GaAs, as reported by Grimsditch, Olego, and Cardona. ${ }^{10}$ This scaled curve agrees well with the LO in-

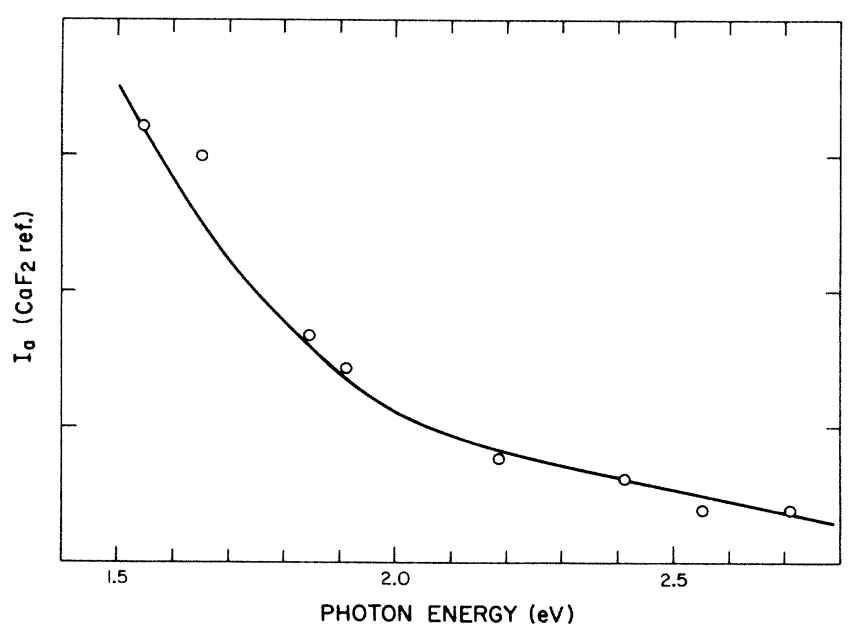

FIG. 3. Intensity of the $a$-GaAs Raman component for the sample of Fig. 2, plotted vs $\hbar \omega_{L}$. The curve shows $d_{\mathrm{OPT}}(\hbar \omega)$. 


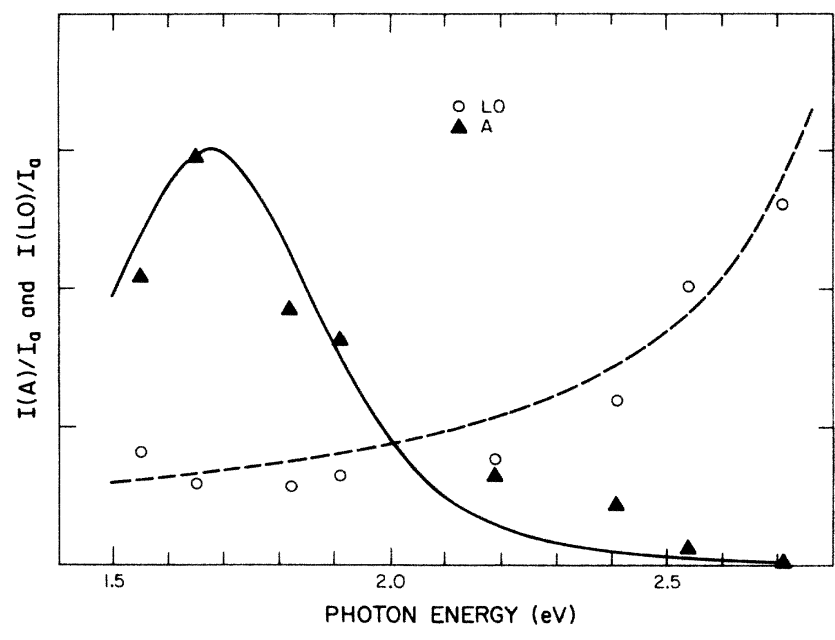

FIG. 4. Comparison of the resonance behavior of the LO line and the $A$ peak, for the implant of Figs. 2 and 3 . The curves are theoretical fits.

tensity data, supporting our $E_{1}$-resonance interpretation.

Figure 4 clearly reveals that the new feature $A$ exhibits a very pronounced resonance, and that this resonance is very different from that exhibited by the LO line. Peak $A$ resonates near $1.7 \mathrm{eV}$ in the red (where it becomes the most prominent feature in the spectrum), unlike the LO line which resonates in the violet, and unlike the $a-\mathrm{GaAs}$ broadband continuum which does not resonate. Peak $A$ is absent from the spectra of both limiting forms, $c$-GaAs and $a-G a A s$. The solid curve in Fig. 4 is a theoretical fit to the data, discussed in Sec. III.

To summarize these experimental results: We have observed a new, strong, low-frequency feature in the Raman spectrum of ion-implanted GaAs having a mixed amorphous-microcrystalline microstructure. This Raman feature has a nearly constant peak position and linewidth. It is not present in either pure $c$-GaAs or pure $a-G a A s$. It is strongly resonant near $1.7 \mathrm{eV}$, in the neighborhood of the $E_{0}$ direct gap of $c$-GaAs, unlike the Raman-allowed LO line which resonates near $E_{1}$. Chemical-etch Raman measurements of the depth profile of peak $A$ show that its strength mirrors that of other measures of ion damage. ${ }^{3,5}$ Also, peak $A$ is not unique to Be-implanted GaAs; we have also observed it in $\mathrm{Si}$-implanted and $\mathrm{SiF}_{3}$-implanted GaAs. $^{5}$

\section{POSSIBLE MODELS FOR THE 47-cm ${ }^{-1}$ PEAK}

The phonon density of states of $c$-GaAs has no structure close to $47 \mathrm{~cm}^{-1},{ }^{11}$ so that the $A$ peak is not due to a disorder-activated crystal feature. Impurity-atom vibrations are ruled out because we have seen it with several implant species $\left(\mathrm{Be}^{+}, \mathrm{Si}^{+}, \mathrm{SiF}_{3}{ }^{+}\right){ }^{5}$ Thus it must be associated with the implantation-damaged $\mathrm{GaAs}$ host. Native defects of $c$-GaAs (interstitials, antisite defects, etc.) remain a possibility. However, when a defect vibration occurs within the fundamental frequency regime of the host $\left(0-300 \mathrm{~cm}^{-1}\right.$ for GaAs), it is drastically frequency broadened because of its admixture with host modes. ${ }^{12}$ Thus, the low frequency and relative sharpness of the $A$ peak are arguments against a native-defect interpretation.

Electronic Raman scattering (ERS) is also unlikely. Acceptor-level ERS has recently been observed in a few high-quality $\mathrm{GaAs}$ crystals at $15 \mathrm{~K}$ using below-band-gap excitation, ${ }^{13}$ special circumstances which differ greatly from our room-temperature above-band-gap experiments on highly damaged GaAs. Though ERS can give rise to a low-frequency band (caused by strain splitting of acceptor ground states), this band is broad, its position is sample dependent, and it is overshadowed by a strong $1 S \rightarrow 2 S$ bound-hole band at higher frequency. ${ }^{13}$ Since our $A$ peak is narrow, sample insensitive, lacks a strong highfrequency partner, and moreover occurs with different dopants, ERS is not the answer.

The low frequency of the $A$ peak suggests the involvement of acoustic phonons and, as discussed below, we believe this to be the case. However, it is not simply due to a size-effect mechanism, such as the phonon-confinement model which well describes the damage-induced downshifting and broadening of the LO line. ${ }^{3,4,14}$ For the LO line, as the crystallite size $L$ decreases the $k$-vector uncertainty $(-1 / L)$ increases to permit participation of phonons in a larger $k$-space volume around the origin. The line broadens and downshifts (Fig. 1) because of the small downward $\omega(k)$ dispersion of the LO branch (see Fig. 1). For acoustic modes, the $\omega(k)$ dispersion near $k=0$ is enormous by comparison, and the corresponding upshift with increasing damage would be very large. This is not observed.

The three mechanisms listed below are, in our view, the most likely candidates for the implantation-induced, infrared-resonant, low-frequency peak: (a) vibrations characteristic of microcrystal-amorphous interface regions; (b) graininess-induced Raman-active acoustic modes, in which the microcrystal-amorphous grainy texture mimics a disordered superlattice; (c) defect-induced Raman-active acoustic modes, in which the microcrystalamorphous interface regions act as extended defects.

We shall briefly describe and criticize these mechanisms. First, it should be noted that (a) and (c) share the feature of a key role played by the interface regions: in (a) as the location of the vibrating atoms, and in (c) as the extended defects which enable defect-assisted Raman scattering by acoustic phonons. Also, (b) and (c) share the feature of acoustic phonons as the vibrational participants in the Raman process. As discussed below, we judge (c) to be the best tentative explanation of the new peak, primarily because it appears to account best for the resonant behavior.

Vibrations characteristic of special atomic-scale configurations at the interfaces between the microcrystals and the amorphous regions of the implanted semiconductor, as invoked in (a), provide a natural explanation of the fact that the $47-\mathrm{cm}^{-1}$ peak occurs only in the mixed microcrystalline-amorphous material, and is absent in both pure phases ( $c$-GaAs and $a-G a A s)$. Also, it would account for a peak whose position is largely invariant to fluence and to photon energy. However, it is difficult to understand the low frequency of $A$ on this basis, as well as 
its resonance.

The idea behind (b) is that the microstructural graininess of the implanted material may structurally imitate a disordered superlattice. Zone-folded longitudinalacoustical (LA) phonons are strongly Raman active in (100)-GaAs $/ \mathrm{Al}_{x} \mathrm{Ga}_{1-x}$ As superlattices. ${ }^{15}$ These LA phonons correspond to $k_{z}=2 \pi / d$ in $c-\mathrm{GaAs}$, where $k_{z}$ is in the stacking direction and $d$ is the superlattice periodicity. These folded acoustic phonons result in sharp, lowfrequency peaks, which resonate just above the band gap. ${ }^{15}$ Similar low-frequency peaks are seen in amorphous superlattices. ${ }^{16}$

For a folded-phonon LA peak at $47 \mathrm{~cm}^{-1}$ in GaAs, the corresponding $d$ value would be $35 \AA .{ }^{15}$ This length is not very different from the $60-\AA$ crystallite size $L$ obtained ${ }^{3}$ for our high-implant sample, suggesting that there may be merit to the idea that graininess mimics a messy superlattice. Also, the resonance behavior of $A$ is similar to that seen for folded phonons. However, since $v \sim d^{-1}$ for folded phonons, model (b) would require the $A$ peak to shift upward with increasing fluence (damage decreases $L$, the analog of $d$ ). This is not observed in Fig. 1 and (unless some mechanism selects a dominant $L$ from the distribution of $L$ 's) presents a serious problem for this model.

Model (c) is a defect-assisted light-scattering mechanism in which the excited electron or hole undergoes two interactions. One is an elastic collision with the "defect," which is here taken to be the crystallite-amorphous inter- face. This results in a momentum transfer producing a breakdown of the $k=0$ selection rule. Acoustic modes may now participate in the other interaction, in which a phonon is created or destroyed. Defect-assisted onephonon Raman scattering has been treated by Menendez and Cardona ${ }^{17}$ and by Berg and $\mathrm{Yu},{ }^{18}$ who show how double-resonance conditions result in large scattering cross sections for phonon wave vectors in special regions within the Brillouin zone.

The solid curve in Fig. 4 represents defect-assisted Raman intensity for acoustic phonons coupled to microcrystal-confined electrons by a deformation potential mechanism. The fit was obtained using a broadened $(0.4$ eV) transition energy of $1.65 \mathrm{eV}{ }^{5}$ (The $E_{0}$ and $E_{0}+\Delta_{0}$ direct band gaps in $c$-GaAs are at 1.42 and $1.76 \mathrm{eV}$.) Double resonance is found to favor acoustic phonons with wave vectors near $0.1 k_{\mathrm{BZ}}$. Fair agreement with the observed $A$-peak resonance is obtained. A detailed treatment of this analysis will be published. ${ }^{5}$

\section{ACKNOWLEDGMENTS}

The authors wish to thank S. Matteson and A. Purdes of the Texas Instruments Central Research Laboratory for the $\mathrm{Be}^{+}$-implanted $\mathrm{GaAs}$ samples, and thank Texas Instruments Incorporated and the Virginia Center for Innovative Technology for supporting this work.
*Present address: Max-Planck-Institut für Festkörperforschung, Stuttgart, Germany.

${ }^{1}$ J. B. Theeten and M. Erman, J. Vac. Sci. Technol. 20, 471 (1982).

${ }^{2}$ D. E. Aspnes, S. M. Kelso, C. G. Olson, and D. W. Lynch, Phys. Rev. Lett. 48, 1863 (1982).

${ }^{3}$ M. Holtz, R. Zallen, O. Brafman, and S. Matteson, Phys. Rev. B (to be published).

${ }^{4}$ K. K. Tiong, P. M. Amirtharaj, F. H. Pollak, and D. E. Aspnes, Appl. Phys. Lett. 44, 122 (1984).

${ }^{5} \mathrm{M}$. Holtz, R. Zallen, and O. Brafman (unpublished).

${ }^{6}$ Careful analysis of the LO component of these spectra reveals no variation of the peak position and linewidth (see Ref. 3).

${ }^{7}$ M-L. Theye and A. Gheorghiu, Sol. Energy Mater. 8, 331 (1982).

${ }^{8}$ D. E. Aspnes and A. A. Studna, Phys. Rev. B 27, 985 (1983).

${ }^{9}$ H. Ehrenreich, H. R. Philipp, and J. C. Phillips, Phys. Rev.
Lett. 8, 59 (1962).

${ }^{10}$ M. H. Grimsditch, D. Olego, and M. Cardona, Phys. Rev. B 20, 1758 (1979).

${ }^{11}$ B. Dorner and D. Strauch (private communication).

${ }^{12}$ A. S. Barker and A. J. Sievers, Rev. Mod. Phys. 47, S1 (1975).

${ }^{13}$ K. Wan and R. Bray, Phys. Rev. B 32, 5265 (1985).

${ }^{14}$ H. Richter, Z. P. Wang, and L. Ley, Solid State Commun. 39, 625 (1981).

${ }^{15}$ C. Colvard, R. Merlin, M. V. Klein, and A. C. Gossard, Phys. Rev. Lett. 45, 289 (1980); C. Colvard, T. A. Gant, M. V. Klein, R. Merlin, R. Fischer, H. Morkoc, and A. C. Gossard, Phys. Rev. B 31, 2080 (1985).

16P. Santos, M. Hundhausen, and L. Ley, Phys. Rev. B 33, 1515 (1986).

${ }^{17}$ J. Menéndez and M. Cardona, Phys. Rev. B 31, 3696 (1985).

${ }^{18}$ R. S. Berg and P. Y. Yu, Phys. Rev. B 35, 2205 (1987). 